\title{
Towards negative cycle canceling in wind farm cable layout optimization
}

\author{
Sascha Gritzbach*, Torsten Ueckerdt, Dorothea Wagner, Franziska Wegner and Matthias Wolf
}

From The 7th DACH+ Conference on Energy Informatics

Oldenburg, Germany. 11-12 October 2018

\author{
*Correspondence: \\ sascha.gritzbach@kit.edu \\ Karlsruhe Institute of Technology, \\ Department of Theoretical \\ Informatics, Karlsruhe, Germany
}

\begin{abstract}
In the WIND FARM CABLING PROBLEM (WCP) the task is to design the internal cabling of a wind farm such that all power from the turbines can be transmitted to the substations and the costs for the cabling are minimized. Cables can be chosen from several available cable types, each of which has a thermal capacity and cost. Until now, solution approaches mainly use MIXED-INTEGER LINEAR PROGRAMS (MILP) or metaheuristics. We present our current state of research on a fast heuristic specifically designed for WCP. We introduce an algorithm that iteratively improves a cable layout by finding and canceling negative cycles in a suitably defined network. Our simulations on publicly available benchmark sets show that the heuristic is not only fast but it tends to produce good results. Currently our algorithm gives better solutions on large wind farms compared to an MILP solver. However, on small to medium instances the solver performs better in terms of solution quality, which represents a starting point for future work.
\end{abstract}

Keywords: Wind farm cable layout, Negative cycle canceling, Network flow, Step function, Heuristic

\section{Introduction}

In view of the European Union's '2030 Energy Strategy', which, among other things, aims at having "at least a 27\% share of renewable energy consumption" (European Commission 2018), renewable energy sources have become increasingly important. In terms of electricity, the gross generation in the EU28 in 2016 came with a 30.2\% share from renewable energy, out of which a $30.9 \%$ share was due to wind energy (European Commission DG ENER Unit A4 2018). WindEurope states that in 2017, additional 15,638 MW of wind power capacitity were installed in the EU28, out of which 3154 MW come from offshore wind farms (WindEurope asbl/vzw 2018).

A typical offshore wind farm consists of turbines and substations. Turbines convert wind energy to electricity which is transported through medium-voltage sea cables, possibly via other turbines, to substations (internal cabling) where the electricity is transformed to the high-voltage level and transported to an onshore grid point (external cabling). 
In the process of planning wind farms, various stages have to be completed. Turbines have to be placed in a way to maximize wind usage and minimize wake effects, substations should be close to the turbines and both the internal and the external cabling need to be found adhering to geographical, economic, and electrical constraints. Ideally, an optimal planning process would unify all stages in a single process.

With the increasing size of newly planned wind farms (e.g., Hornsea Project Three may include up to 300 turbines (Hornsea Project Three Offshore Wind Farm 2018)), planning by hand becomes more difficult and hence automated approaches become more desirable. Automated approaches, however, tend to have difficulties with the complexity of a unified planning process (Santos et al. 2014), which leads to considering subsets of the planning stages separately.

The cost for the internal cabling accounts for approximately $17 \%$ of the total cost for planning and building a wind farm (Santos et al. 2014). Therefore, it is essential to find a cost-efficient cabling. When designing the internal cabling isolatedly, the positions of turbines and substations are considered fixed and grid points and high-voltage cabling are not of interest at this time. Also as an input to the problem, there are given possible connections between turbines and between turbines and substations. These connections can be used for routing the electricity produced by the turbines. Furthermore, there is a set of possible cable types that can be installed on the connections in order to transmit electricity. Each cable type has a thermal capacity and a cost per unit length for material and installation. The goal of this planning stage is to identify connections on which electricity is routed and to assign a cable type to every connection such that all electricity can be transported to substations. We call this planning stage the WIND FARM CABLING Problem (WCP). Since WCP includes the NP-hard problem CapaCitated Minimum Spanning Tree (Cerveira et al. 2014), it is NP-hard as well.

Contribution and Outline We present a basic implementation of a heuristic for WCP, which first computes a feasible solution and iteratively improves it by finding and canceling negative cycles in a suitably defined graph. To find these negative cycles we use a slight modification of the Bellman-Ford Algorithm (Bellman 1958; Ford et al. 2010). Evaluating the heuristic on the wind farm benchmark sets presented by Lehmann et al. (2017) shows that it runs fast and gives good results compared to the solution computed by the MILP solver Gurobi (Gurobi optimizer reference manual 2018) within one hour.

In the following section we review the literature on the WIND FARM CABLING PROBLEM (WCP), applications of negative cycle canceling, and other related problems. We model WCP formally in the "Model" section. In the "Algorithmic overview" section, we explain our heuristic in detail. We report and discuss the results of the simulation of our heuristic in the "Simulations" section and conclude with a thorough overview of possible research directions.

\section{Related work}

Since the integrated planning process which includes turbine and substation placement as well as internal and external cabling comes with a level of complexity that is hard to manage, often a single planning step at a time is considered for optimization in the literature. 
For finding the optimal cable layout between turbines and substations with fixed positions-which is also the scope of this work-one of the first papers was by Berzan et al. (2016), in which they propose a hierarchical decomposition of the problem into several layers. They use well-studied graph problems to solve the so-called Circuit and Substation layers, in which only one substation is considered at a time, when there is only one cable type available.

Since then, various approaches have been taken for more elaborate problems considering different optimization functions and sets of constraints. With the high complexity of the problem in mind, metaheuristics, such as Genetic Algorithms (Zhao et al. 2004; Shirshak et al. 2017; Dahmani et al. 2015) or Simulated Annealing, (Lehmann et al. 2017) are very popular. While these approaches do not guarantee provably optimal solutions, they are able to provide good solutions within short running times. To the contrary, exact solutions can be provided by INTEger Linear Program (ILP) or Mixed-INTEger Linear PROGRAM (MILP) formulations, which need more time and therefore only work on small instances. Lumbreras and Ramos (2013), for example, consider losses along branches, stochasticity in wind inputs and component failures in an ILP and Cerveira et al. (2014) use a graph-theoretic flow model on wind farms with a single substation and use the resemblance to the CApacitated Minimum Spanning Tree (CMST). Based on the flow model, they include constraints representing the CMST into an MILP formulation.

In our work, we use a flow model similar to the one presented by Cerveira et al. (2014) representing how turbine production is routed to one of multiple substations. We aim at finding a flow of minimum cabling cost and apply a well-known technique from network flow theory called negative cycle canceling. Negative cycle canceling was first proposed in the context of finding minimum cost circulations in flow networks (Klein 1967). Goldberg and Tarjan (1989) achieve a strongly polynomial running time for a cycle-canceling-based algorithm for the minimum cost flow by suitably choosing the cycles to cancel. The bound for the running time of this algorithm was later tightened by Radzik and Goldberg (1994).

Ouorou and Mahey (2000) employ negative cycle canceling to solve the Minimum Multicommodity Flow Problem with nonlinear cost functions. Negative cycle canceling is also used in combination with tabu search to tackle the Capacity Expansion Problem for multicommodity flow networks (de Souza et al. 2008), which can be modeled as a Multicommodity Flow Problem with non-convex and non-smooth cost functions.

Optimization problems that are similar to WCP appear for example in logistics. In the Single-Sink Edge Installation Problem introduced by Salman et al. (2001) the production of multiple sources must be transported to a single sink. On every connection a mixture of various cable types (including multiple copies of the same type) needs to be installed such that the cables provide sufficient capacity. Similarly, in the Buy-at-Bulk Problem (see (Gupta and Könemann 2011)), the cost of routing flow along a connection is given by a concave function representing economies of scale. In both cases, the amount of flow on a single connection is unlimited.

One of the main characteristics of our problem is the step cost function with an upper limit on every connection. Gabrel et al. (1999) consider similar step cost functions in a Multicommodity Flow Problem and provide a method for finding exact solutions using a specialized Bender's Decomposition procedure. The exact solutions, however, come at the price of only being able to solve small instances with up to 20 vertices in reasonable time. 
Our approach, on the other hand, has been tested on instances with up to 500 vertices providing good solutions within 50 seconds on average and 7.5 minutes in the worst case.

\section{Model}

In this section, we formalize the WIND FARM CABLING Problem (WCP). We understand turbines and substations as vertices of a graph $G=(V, E)$, i.e., if $V_{T}$ and $V_{S}$ denote the sets of turbines and substations, respectively, then $V=V_{T} \cup V_{S}$ and $V_{T} \cap V_{S}=\emptyset$. While the direction of a connection between a turbine and a substation or between two turbines does not matter in the real world, for the sake of modeling we impose an arbitrary direction on every connection. This implies that $G$ is a directed graph, i.e., for every edge $e$ there are vertices $u$ and $v$ such that $e=(u, v)$ and we say $e$ goes from $u$ to $v$. We assume that turbine production that is transmitted to a substation is transmitted into the connection to the grid point. In particular, it is not routed to a second substation first. To simplify the description of our algorithm, we therefore assume that there are no edges connecting two substations. Moreover, we assume that each turbine has a standardized production of one unit and each substation has a capacity modeled by a function cap sub $V_{S} \rightarrow \mathbb{N}$. Additionally, each edge is assigned a positive length by the function len: $E \rightarrow \mathbb{R}_{>0}$, which represents the geographic distance between the endpoints of the edge.

Along each edge we may place a single cable, whose type is chosen from a finite set of cable types. Each cable type is uniquely determined by its capacity cap ${ }_{\text {cab }} \in \mathbb{N}$ and its cost per unit length $\mathrm{c}_{\mathrm{cab}} \in \mathbb{R}_{\geq 0}$. We therefore identify each cable type with the pair ( cap $_{\mathrm{cab}}, \mathrm{c}_{\mathrm{cab}}$ ) and define the set $K$ of all allowed cable types represented by these pairs. For the ease of representation we assume that $K$ contains the two special cable types $(0,0)$ and $(\infty, \infty)$ called trivial cable types. The former represents the case that no cable is built along an edge and the latter the case that no cable has sufficient capacity. Based on the cable types we define a cost function c: $\mathbb{Z} \rightarrow \mathbb{R}_{\geq 0} \cup\{\infty\}$ by

$$
\mathrm{c}(x)=\min \left\{\mathrm{c}_{\mathrm{cab}}:\left(\mathrm{cap}_{\mathrm{cab}}, \mathrm{c}_{\mathrm{cab}}\right) \in K,|x| \leq \mathrm{cap}_{\mathrm{cab}}\right\} \quad \forall x \in \mathbb{Z},
$$

i.e., we choose the cheapest cable type that has sufficient capacity to transport $|x|$ units of flow.

In total, a wind farm is then modeled as a network $\mathcal{N}=\left(G, V_{T}, V_{S}\right.$, len, cap sub, $\left.\mathrm{c}\right)$. The network incorporates turbines $V_{T}$, substations $V_{S}$ with a capacity cap sub each, and connections between turbines and substations described by the graph $G$, as well as the length of the connections len and costs per length c for using the connections. Note that we do not explicitly include the set of cable types $K$ as all necessary information on them is incorporated in the function c.

A flow in the network $\mathcal{N}$ is a function $f: E \rightarrow \mathbb{R}$. Since we imposed an arbitrary direction on each edge, we are able to identify the direction of a flow on an edge. More formally, if $f(u, v)>0$ (resp. $<0)$ for some edge $(u, v)$, we interpret that as $f(u, v)$ (resp. $-f(u, v))$ units flowing from $\mathrm{u}$ to $\mathrm{v}$ (resp. from $\mathrm{v}$ to $\mathrm{u}$ ). For every vertex $\mathrm{u}$ we define its net flow by $f_{\text {net }}(u)=\sum_{(w, u) \in E} f(w, u)-\sum_{(u, w) \in E} f(u, w)$. A flow $\mathrm{f}$ is feasible if it satisfies the conservation of flow for both turbines (Eq. 2) and substations (Eq. 3) and if there is no outflow at any substation (Eq. 4). Recall that we assume a standardized production of one unit at each turbine. 


$$
\begin{array}{lrl}
f_{\text {net }}(u)=-1 & \forall u \in V_{T}, \\
f_{\text {net }}(u) \leq \operatorname{cap}_{\text {sub }}(u) & \forall u \in V_{S}, \\
f(u, v) \geq 0 & \forall v \in V_{S}, \forall(u, v),(v, w) \in E \\
f(v, w) \leq 0 &
\end{array}
$$

The costs of a feasible flow $f$ are computed as the sum of the individual costs of every edge.

$$
\operatorname{cost}(\mathcal{N}, f)=\sum_{e \in E} \mathrm{c}(f(e)) \cdot \operatorname{len}(e) .
$$

The goal of WCP is to find a feasible flow $f$ with minimum costs. Hence, it can be summarized as follows.

Wind Farm Cabling Problem WCP

Instance: A network $\mathcal{N}=\left(G, V_{T}, V_{S}\right.$, len, cap sub $\left._{1}, \mathrm{c}\right)$.

Objective: Find a feasible flow $f$ on $G$ such that $\operatorname{cost}(\mathcal{N}, f)$ is minimum among all choices of feasible flows.

\section{Negative cycle algorithm}

In this section, we describe an approach of finding and canceling negative cycles in order to solve WCP heuristically. The main idea of our heuristic is to repeatedly set up a residual graph from a flow, finding a negative cycle, and cancel negative cycles in the residual graph. Every cancellation yields a better solution to WCP. In the first part, we give an overview of our heuristic. Whereas in the second part, we describe the components used in the heuristic in more detail.

\section{Algorithmic overview}

Before we describe the algorithm, we introduce essential graph theoretical terms. We define a walk from $u$ to $w$ as a sequence of-not necessarily distinct-edges $\left(\left(u, v_{1}\right)=\right.$ : $\left.e_{0}, e_{1}, \ldots, e_{k}:=\left(v_{k}, w\right)\right)$ such that the end vertex of $e_{i-1}$ is the same as the start vertex of $e_{i}$ for $i \in\{1, \ldots, k\}$. A walk is closed if $u=w$ and it is side-trip free if $e_{i}$ is not the reverse edge of $e_{i-1}$ for all $i \in\{1, \ldots, k\}$, i.e., the walk does not contain a closed subwalk of length 2 . Closed walks where the end vertices of all edges are distinct are called cycles.

Given a wind farm $\mathcal{N}$ we first compute an initial feasible flow $f$ (Lines 2-4; all line references in this section refer to Algorithm 1). For each turbine $u \in V_{T}$ we perform a breadth-first search from $u$ ignoring all edges and substations without free capacity. When the search finds a substation for the first time, the flow on the path from $u$ to the substation is increased by 1 . Starting with this initial flow, we iteratively identify simple changes of the flow that decrease the costs.

In each iteration of the heuristic, we set up the residual graph $R$ (Line 8), which we define as follows. We denote the underlying directed graph of the wind farm $\mathcal{N}$ by $\mathrm{G}$ as defined in the "Model" section. We set $V(R)=V(G) \cup\{s\}$ and $E(R)=E(G) \cup\{(u, v)$ : $(v, u) \in E(G)\} \cup\left\{(u, s),(s, u): u \in V_{S}\right\}$, where $s$ is a special vertex representing a super substation. 


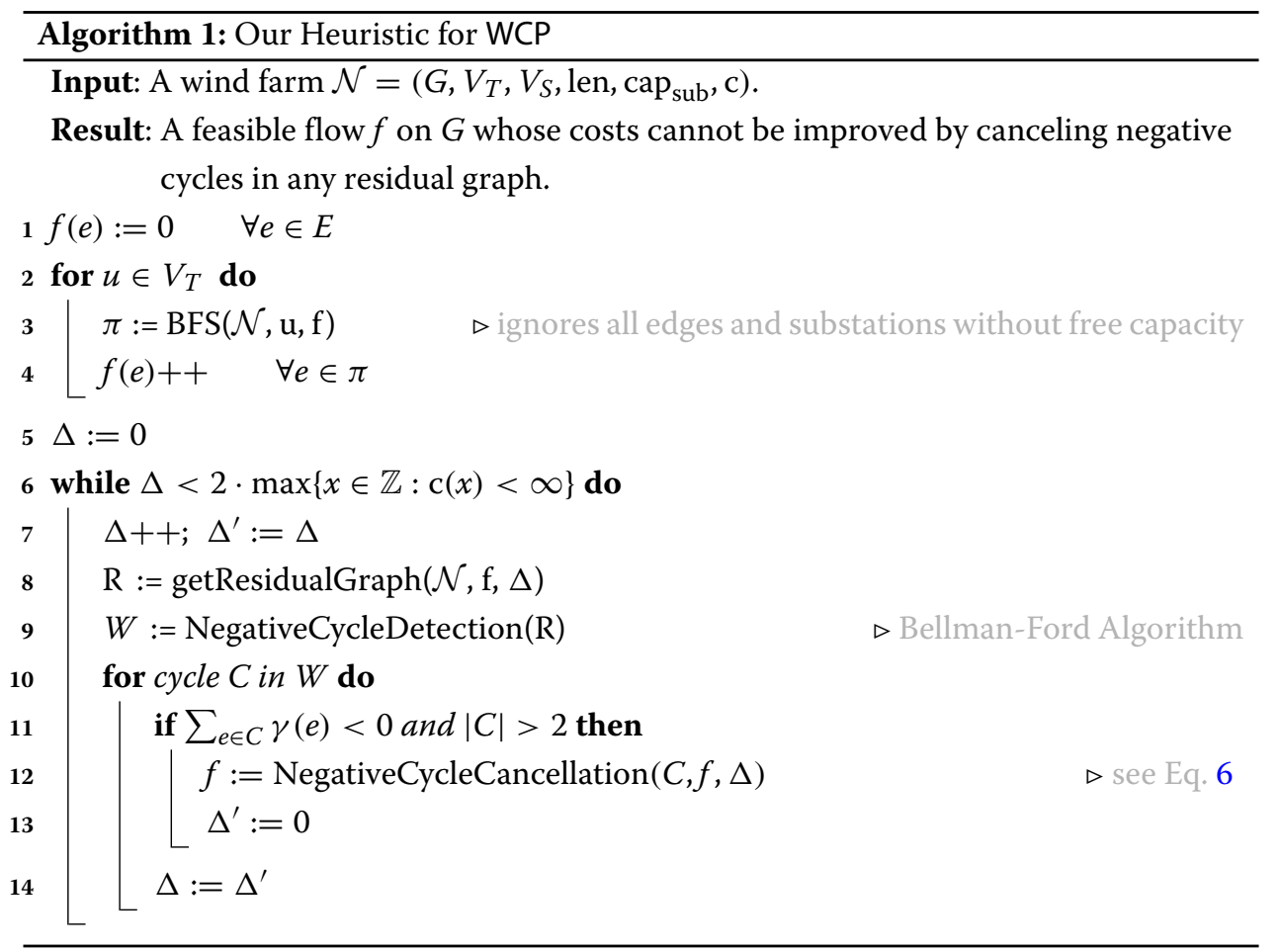

Let $f$ be the flow computed in the previous iteration and $\Delta \in \mathbb{N}$ with initialization shown in Lines 5 and 7. In addition, we define the cost function $\gamma: E(R) \rightarrow \mathbb{R}$ as explained below. We then search for a closed side-trip-free walk with negative total costs in $\mathrm{R}$ (Line 9). To this end, we use a slight adaptation of the Bellman-Ford Algorithm. If there is no such walk, we increment $\Delta$ and set up a new residual graph. Otherwise, if there is such a walk $W$ in $R$, we split $W$ into cycles $C_{1}, \ldots, C_{l}$. Figure 1 shows an example of this decomposition into cycles. We check for each cycle $C_{i}$ whether its total costs are negative and whether $C_{i}$ has length at least 3 (Line 11). If both conditions hold, we cancel $C_{i}$ (see Eq. 6 and Line 12). Note that the cost function $\gamma$ is defined in such a way that cycles that decrease the cost have negative total costs. We cancel $C$ by changing the flow $f$ by $\Delta$ along $C$. More formally, we define a new flow $f^{\prime}$ for all $(u, v) \in E(G)$ by

$$
f^{\prime}(u, v)= \begin{cases}f(u, v)+\Delta, & \text { if }(u, v) \in E(C), \\ f(u, v)-\Delta, & \text { if }(v, u) \in E(C), \\ f(u, v), & \text { if }(u, v),(v, u) \notin E(C) .\end{cases}
$$

Here, $E(C)$ denotes the set of edges that form the cycle $C$; see Fig. 2 for an example of canceling a cycle.

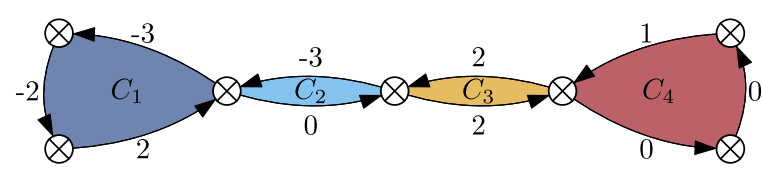

Fig. 1 Decomposing a side-trip-free walk into cycles. A side-trip-free closed walk $W$ with total costs -1 . It can be decomposed into four cycles $C_{1}, \ldots, C_{4}$. Two of them $\left(C_{2}\right.$ and $\left.C_{3}\right)$ consist of two edges and are discarded. Of the other two, $C_{4}$ has positive total costs and is discarded as well. The remaining cycle $C_{1}$ has total costs -2 and is canceled 
Note that if a cycle has length exactly 2 , it consists of an edge $(u, v)$ and its reverse $(v, u)$. Hence, canceling it means sending $\Delta$ units in both directions specified by $(u, v)$ and $(v, u)$, which does not change the flow. Canceling it would result in an infinite loop in Line 10.

Finally, if at least one cycle $C_{i}$ in $W$ was canceled, we reset $\Delta$ to 1 and build a new residual graph based on the new flow after the cycle cancellation in the current iteration. The heuristic terminates once it does not cancel a negative cycle in the residual graphs of the currently cheapest feasible flow for all values of $\Delta$.

\section{The residual costs}

The description above assumes a cost function $\gamma$ on the residual graph $R$ such that negative cycles in $R$ correspond to cycles that decrease the costs of the flow if they are canceled. We define $\gamma$ in this section.

Let $f$ be a feasible flow in $\mathcal{N}$ and $\Delta \in \mathbb{N}$. We define $\gamma: E(R) \rightarrow \mathbb{R} \cup\{\infty\}$ such that canceling a cycle with negative costs, i.e., a cycle $C$ with $\sum_{e \in E(C)} \gamma(e)<0$, preserves feasibility and reduces the total costs. Consider an edge $(u, v) \in E(G)$. Intuitively, $\gamma(u, v)$ represents the change of costs for the original flow $f(u, v)$ if additional $\Delta$ units are sent from $u$ to $v$. More formally, if both $u$ and $v$ are turbines, the costs of the corresponding edges in the residual network $R$ are defined by

$$
\begin{aligned}
& \gamma(u, v)=(\mathrm{c}(f(u, v)+\Delta)-\mathrm{c}(f(u, v))) \cdot \operatorname{len}(u, v), \\
& \gamma(v, u)=(\mathrm{c}(f(u, v)-\Delta)-\mathrm{c}(f(u, v))) \cdot \operatorname{len}(u, v) .
\end{aligned}
$$

Note that by this definition if $f(u, v)+\Delta$ or $-f(v, u)+\Delta$ exceeds the largest non-trivial cable capacity, we define $\gamma(u, v)=\infty$ or $\gamma(v, u)=\infty$, respectively. If one of the vertices, say $v$, is a substation, we define the costs in the same way unless $f(u, v)<\Delta$. In this case, we define $\gamma(v, u)=\infty$ to ensure that no flow leaves the substation (see Eq. 4). Therefore, we have $\gamma\left(v_{1}, u_{1}\right)=\infty$ in Fig. 2. For the edges between a substation $w$ and the super substation $s$ we define $\gamma(w, s)=0$ if $f_{\text {net }}(w)+\Delta \leq \operatorname{cap}_{\text {sub }}(w)$, and $\gamma(w, s)=\infty$ otherwise.

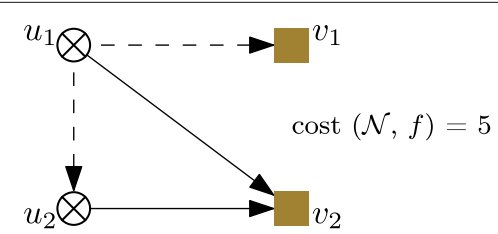

(a)

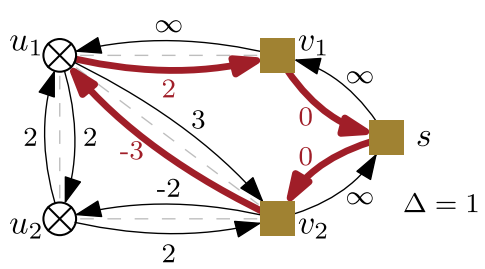

(c)

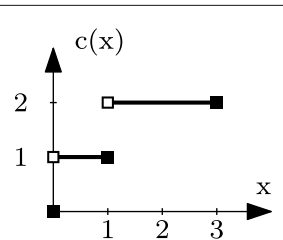

(b)

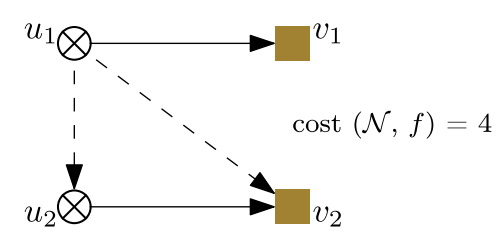

(d)

Fig. 2 Canceling a negative cycle. (a) A feasible flow $f$ on the network $\mathcal{N}$ is shown. Turbines are represented by circles with a cross and substations by filled squares. All substations have capacity 2 . The edge $\left(u_{1}, v_{2}\right)$ has length 3, all others have length 2. Dashed lines represent edges without flow, regular edges have a flow of 1 . (b) The cost function c induced by the cable types $(0,0),(1,1),(3,2)$, and $(\infty, \infty)$. (c) The residual graph $R$ based on $f$ and $\Delta=1$. The fat red edges mark a negative cycle. (d) The new flow after canceling the negative cycle 
This ensures that the substation capacity will not be exceeded (see Eq. 3). In Fig. 2 the substation $v_{2}$ has reached its capacity and hence we set $\gamma\left(v_{2}, s\right)=\infty$. For the reverse edge, we set $\gamma(s, w)=0$ if $\Delta \leq f_{\text {net }}(w)$ and $\gamma(s, w)=\infty$ otherwise, which makes sure that $w$ has a non-negative net flow. As $v_{1}$ has no incoming flow in Fig. 2, the residual cost $\gamma\left(s, v_{1}\right)$ is set to $\infty$.

Clearly, the cost function $\gamma$ on $R$ can have negative values since it is possible that after a change of flow by $\Delta$ a cheaper cable type suffices for the new flow. Hence, cycles of negative total costs can exist in $R$. By the definition of $\gamma$, it holds for any cycle $C$ in $R$ with finite costs that the flow $f^{\prime}$ obtained from $f$ by canceling $C$ is feasible. Moreover, we have

$$
\operatorname{cost}\left(\mathcal{N}, f^{\prime}\right)=\operatorname{cost}(\mathcal{N}, f)+\sum_{e \in E(C)} \gamma(e)
$$

Hence, if $C$ has negative total costs, $f^{\prime}$ incurs less cost than the previous flow $f$.

\section{Simulations}

In this paper, we introduce a heuristic that is able to calculate feasible solutions for WCP in milliseconds and thus, it provides an alternative to MILPs, even though our algorithm may not solve the problem to optimality. In this section, we evaluate the solution quality and running time of our heuristic and compare it to the baseline MILP which minimizes the step cost function in Eq. 5 subject to the constraints given by Eqs. 2-4. We analyze the solution quality and performance on the benchmark sets published by Lehmann et al. (2017) using different criteria namely the number of turbines $\left|V_{T}\right|$ (Fig. 3a), and the benchmark sets $\mathcal{N}_{i}$ with $1 \leq i \leq 4$ (Fig. 3b). The benchmark sets include data on cables and their characteristics.

We calculate the baseline by the MILP using Gurobi 7.0.2 (Gurobi optimizer reference manual 2018). Our code is written in $\mathrm{C}++14$; compiled with GCC 7.3.1 using the -03 - march=native flags. The simulations run on a 64-bit architecture with four 12-core CPUs of AMD 6172 clocked at $2.1 \mathrm{GHz}$ with $256 \mathrm{~GB}$ RAM running OpenSUSE 42.3. Though we have a multi-core machine, we run all simulations-including the MILPin single-threaded mode to ensure comparability. In addition, for the simulations with regards to the MILP we opt for a quantity measurement (similar to (Lehmann et al. 2017)). That means we run a large fraction of benchmark instances with a time limit of one

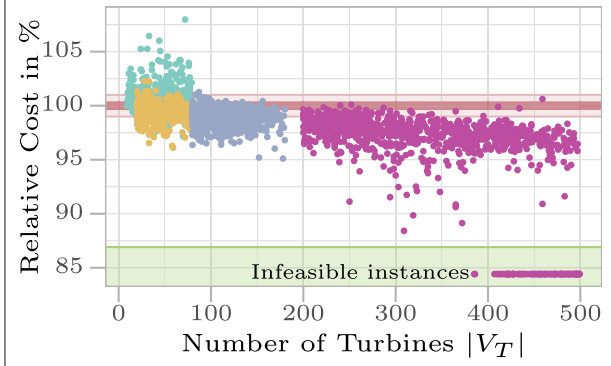

(a)

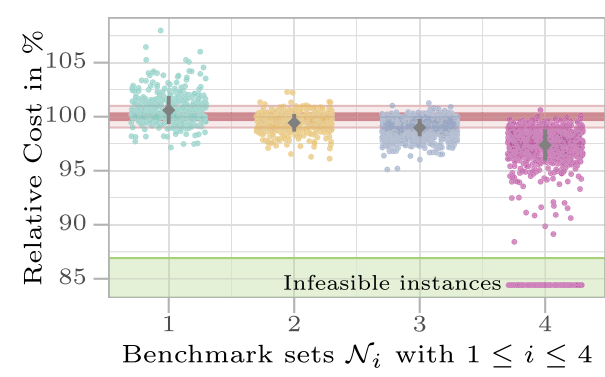

(b)

Fig. 3 Evaluation of the Negative Cycle Algorithm using different criteria: (a) number of turbines $\left|V_{T}\right|$, and (b) benchmark sets $\mathcal{N}_{i}$ with $1 \leq i \leq 4$. We compare all criteria with the relative cost-meaning the ratio of the best solution found by our algorithm to the best solution found by Gurobi. Some instances are identified as infeasible (green marked range) 
hour for every instance instead of running a few instances for a longer time and solving them-if possible - to optimality. This provides us with a broader range of results.

The small benchmark sets $\mathcal{N}_{1}$ and $\mathcal{N}_{2}$ consist of 500 instances each, each of which has 10 to 80 turbines. The instances of $\mathcal{N}_{1}$ have exactly one substation each and $\mathcal{N}_{2}$-instances have two to seven substations. For these benchmark sets our algorithm finds solutions that are at least as good as the MILP within one hour in $47.2 \%$ and $80.7 \%$ of the cases (see Fig. 3b). However, our algorithm finds the solutions in milliseconds (see Table 1). The benchmark sets $\mathcal{N}_{3}$ and $\mathcal{N}_{4}$ each have 1000 instances. The instances of $\mathcal{N}_{3}$ are mediumsized with 80 to 200 turbines and four to ten substations each, whereas the instances in $\mathcal{N}_{4}$ are large-sized with 200 to 1000 turbines and 10 to 40 substations. Here, our algorithm performs much better than the MILP within one hour. In $93.6 \%$ and $99.6 \%$ of the instances of $\mathcal{N}_{3}$ and $\mathcal{N}_{4}$, respectively, our algorithm finds solutions that are at least as good as the MILP within one hour (see Fig. 3b). From Figs. $3 \mathrm{a}$ and $3 \mathrm{~b}$ we can see that our algorithm is mostly outperformed by the MILP on instances with small number of turbines and with a small number of substations.

Summarizing the evaluation, the Negative Cycle Algorithm is a good alternative especially when it comes to large instances. For small instances there is room for improvement, e.g., by analyzing cases where the MILP is better.

\section{Conclusion and future work}

Based on canceling negative cycles we present a heuristic for the WIND FARM CABLING PROBLEM (WCP). It runs very quickly-in the order of milliseconds to a few minutes depending on the size of the wind farm given as input-and provides very good results. Our comparison to the solutions of an MILP solver after one hour indicates that our heuristic often produces better solutions even though it takes only a fraction of the time.

Moving forward in this research in progress, we want to continue identifying strengths and weaknesses of our heuristic by running further analysis on the data provided by our simulations and by elaborating on the correctness of our algorithm. We plan to conduct further simulations on the MILP-side with longer running times of several days or even weeks. Furthermore, we hope we are able to compare our heuristic to other (meta-)heuristics tackling WCP. All of these will help us improve our algorithmic approach to solving WCP by canceling negative cycles.

So far, in all our simulations we only considered one set of cable types. It would be interesting to run our algorithm for different cable types and compare the findings depending on the characteristics of various cable type sets. We also assume standardized turbine production throughout the wind farm. We hope we are able to account for non-uniform productions by adjusting the residual graph so that the non-uniform net-flow at turbines is maintained.

Table 1 Performance indicators of the Negative Cycle Algorithm, where Columns 2 to 4 represent numbers of iterations and Columns 5 to 7 show the running times in milliseconds

\begin{tabular}{|c|c|c|c|c|c|c|}
\hline \multirow[t]{2}{*}{$\overline{\mathcal{N}_{i}}$} & \multicolumn{3}{|c|}{ Iterations } & \multicolumn{3}{|c|}{ Time in $\mathrm{ms}$} \\
\hline & (min) & (avg) & $(\max )$ & $(\min )$ & (avg) & (max) \\
\hline 1 & 8 & 41.20 & 143 & 0.72 & 40.63 & 293.42 \\
\hline 2 & 17 & 49.97 & 106 & 3.77 & 51.72 & 220.92 \\
\hline 3 & 71 & 124.87 & 235 & 157.69 & 575.02 & 2968.56 \\
\hline 4 & 189 & 376.27 & 638 & 2815.12 & $149,209.92$ & $440,235.07$ \\
\hline
\end{tabular}


Those instances, for which the MILP provides better solutions, show that there are nonoptimal feasible flows that are not further improvable by our algorithm. Such a flow can be seen as a local minimum for our heuristic. To escape these local minima there are multiple viable strategies. One way could be to allow changes that temporarily increase the total costs, e.g., by canceling cycles with small positive total costs if no negative cycle is found-similar to metaheuristics like Simulated Annealing. As another approach we could search for more complex circulations with negative total costs in the residual graph, e.g., two cycles that share an edge, and cancel those. From a more theoretical standpoint, it would be very interesting to see if optimality can be achieved by identifying only a small set of more complex circulations.

So far, we restricted our heuristic to a single initialization strategy, namely breadth-first search. Other techniques might influence the trajectory of canceling negative cycles and therefore our heuristic might converge to other local minima.

Since our heuristic finds good solution within a short period of time, it might be interesting to see how those solutions can help the MILP to solve WCP. More specifically, a solution given by our algorithm can be given to the solver as an initial feasible solution from which the optimization procedure can be started (warm start). Then, the performances of the MILP with warm and with cold start can be compared in further simulations.

In our model, it is not required that every turbine has only one edge with outgoing flow. When applying AC-flow or its DC-approximation including phase angles at vertices, it might be desirable to prohibit splitting flow at vertices. In the existing literature, requiring unsplittable flow is often neglected to reduce the complexity of the problem. In terms of future work, allowing only one edge per turbine with outgoing flow in our heuristic seems to be possible by suitably modifying the residual graph and the residual costs. With that, we hope that our model represents real-world wind farms more realistically.

\title{
Abbreviations
}

AC: Alternating current; CMST: CAPACITATED MInImUM SPANNING TREE; DC: Direct current; EU28: the 28 member countries of the European Union; ILP: INTEGER LINEAR PROGRAm; MILP: MIXED-INTEGER LINEAR Program; WCP: WIND FARM CABLING PROBLEM

\section{Funding}

This work was funded (in part) by the Helmholtz Program Storage and Cross-linked Infrastructures, Topic 6 Superconductivity, Networks and System Integration, and by the Helmholtz future topic Energy Systems Integration. Publication costs for this article were sponsored by the Smart Energy Showcases - Digital Agenda for the Energy Transition (SINTEG) programme.

\section{Availability of data and materials}

Benchmark sets are publicly available as stated in Lehmann et al. (2017).

\author{
About this Supplement \\ This article has been published as part of Energy Informatics Volume 1 Supplement 1, 2018: Proceedings of the 7th \\ $\mathrm{DACH}+$ Conference on Energy Informatics. The full contents of the supplement are available online at https:// \\ energyinformatics.springeropen.com/articles/supplements/volume-1-supplement-1.
}

\section{Authors' contributions}

DW introduced the topic of wind farm layout and provided overall supervision and guidance. TU contributed general ideas and theoretical insights on negative cycle canceling. SG, FW, and MW implemented and evaluated the algorithms and wrote the article. All authors discussed the results and commented on the manuscript. All authors have approved the final text.

\section{Competing interests}

The authors declare that they have no competing interests.

\section{Publisher's Note}

Springer Nature remains neutral with regard to jurisdictional claims in published maps and institutional affiliations. 


\section{Published: 10 October 2018}

\section{References}

Berzan C, Veeramachaneni K, McDermott J, O'Reilly U (2016) Algorithms for cable network design on large-scale wind farms. http://thirld.com/files/msrp_techreport.pdf. Accessed 4 Jan 2016

Bellman R (1958) On a routing problem. Q Appl Math 16:87-90. https://doi.org/10.1090/qam/102435

Cerveira A, Baptista J, Solteiro Pires EJ (2014) Optimization design in wind farm distribution network. In: Herrero Á, Baruque B, Klett F, Abraham A, Snášel V, de Carvalho CPLFA, Bringas PG, Zelinka I, Quintián H, Corchado E (eds). International Joint Conference SOCO'13-CISIS'13-ICEUTE'13. Springer International Publishing. ISBN 978-3-319-01854-6, Cham. pp 109-119

de Souza MC, Mahey P, Gendron B (2008) Cycle-based algorithms for multicommodity network flow problems with separable piecewise convex costs. Networks 51(2):133-141. https://doi.org/10.1002/net.20208

Dahmani O, Bourguet S, Machmoum M, Guerin P, Rhein P, Josse L (2015) Optimization of the connection topology of an offshore wind farm network. IEEE Syst J 9:1519-1528

European Commission (2018) 2030 Energy Strategy. https:/ec.europa.eu/energy/en/topics/energy-strategy-andenergy-union/2030-energy-strategy. Accessed 31 May 2018

European Commission DG ENER Unit A4 (2018) Energy datasheets: EU28 countries. https://ec.europa.eu/energy/sites/ ener/files/documents/countrydatasheets_feb2018.xlsx. Accessed 31 May 2018

Ford LR, Fulkerson Jr., Fulkerson DR (2010) Flows in Networks. Princeton University Press, New Jersey

Gabrel V, Knippel A, Minoux M (1999) Exact solution of multicommodity network optimization problems with general step cost functions. Oper Res Lett 25(1):15-23. ISSN 0167-6377. https://doi.org/10.1016/S0167-6377(99)00020-6

Goldberg AV, Tarjan RE (1989) Finding minimum-cost circulations by canceling negative cycles. J ACM 36(4):873-886. ISSN 0004-5411. https://doi.org/10.1145/76359.76368

Gupta A, Könemann J (2011) Approximation algorithms for network design: A survey. Sur Oper Res Manag Sci 16(1):3-20. ISSN 1876-7354. https://doi.org/10.1016/j.sorms.2010.06.001

Gurobi optimizer reference manual (2018) Gurobi Optimization, Inc. http://www.gurobi.com. Accessed 31 May 2018

Hornsea Project Three Offshore Wind Farm (2018) 4C Offshore Ltd. https://www.4coffshore.com/windfarms/hornseaproject-three-united-kingdom-uk 1 k.html. Accessed 31 May 2018

Klein M (1967) A primal method for minimal cost flows with applications to the assignment and transportation problems. Manag Sci 14(3):205-220. https://doi.org/10.1287/mnsc.14.3.205

Lehmann S, Rutter I, Wagner D, Wegner F (2017) A simulated-annealing-based approach for wind farm cabling. In: Proceedings of the Eighth International Conference on Future Energy Systems, e-Energy '17. ISBN 978-1-4503-5036-5. ACM, New York. pp 203-215. https://doi.org/10.1145/3077839.3077843

Lumbreras S, Ramos A (2013) Optimal design of the electrical layout of an offshore wind farm applying decomposition strategies. IEEE Transactions on Power Systems 28(2):1434-1441. ISSN 0885-8950. https://doi.org/10.1109/TPWRS. 2012.2204906

Ouorou A, Mahey P (2000) A minimum mean cycle cancelling method for nonlinear multicommodity flow problems. Eur J Oper Res 121(3):532-548. ISSN 0377-2217. https://doi.org/10.1016/50377-2217(99)00050-8

Radzik T, Goldberg AV (1994) Tight bounds on the number of minimum-mean cycle cancellations and related results. Algorithmica 11(3):226-242. ISSN 1432-0541. https://doi.org/10.1007/BF01240734

Salman FS, Cheriyan J, Ravi R, Subramanian S (2001) Approximating the single-sink link-installation problem in network design. SIAM J Optim 11(3):595-610. https://doi.org/10.1137/S1052623497321432

Santos VP, António Sarmento JNA, Alves M (2014) Offshore wind farm layout optimization - state of the art. J Ocean Wind Energy 1(1):23-29

Shirshak K, Nandigam D, Nandigam M (2017) Design of electrical layout of offshore wind farms. J Renew Sust Energ 9(4):043303. https://doi.org/10.1063/1.4995272

WindEurope asbl/vzw (2018) Wind in power 2017. https://windeurope.org/wp-content/uploads/files/about-wind/ statistics/WindEurope-Annual-Statistics-2017.pdf. Accessed 31 May 2018

Zhao M, Chen Z, Blaabjerg F (2004) Optimization of electrical system for a large DC offshore wind farm by genetic algorithm. In: Proceedings of NORPIE 2004. EPE Association, Brussels. pp 1-8

\section{Submit your manuscript to a SpringerOpen ${ }^{\circ}$ journal and benefit from:}

> Convenient online submission

- Rigorous peer review

- Immediate publication on acceptance

- Open access: articles freely available online

- High visibility within the field

- Retaining the copyright to your article

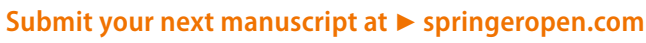

